\title{
Interaction between ICAM1 in endothelial cells and LFA1 in $T$ cells during the pathogenesis of experimental Parkinson's disease
}

\author{
WENJIE LI ${ }^{1,2}$, SHENG CHEN $^{3}$, YUAN LUO $^{2}$, YEZI XIA ${ }^{2}$, QIANQIAN MA ${ }^{2}$, QI YAO $^{2 *}$ and JIANQING WU ${ }^{1^{*}}$ \\ ${ }^{1}$ Department of Geriatrics, Jiangsu Provincial Key Laboratory of Geriatrics, First Affiliated Hospital of \\ Nanjing Medical University, Nanjing, Jiangsu 210000; ${ }^{2}$ Department of Geriatrics, Ningbo First Hospital; \\ ${ }^{3}$ Department of Nephrology, Ningbo Medical Center Lihuili Hospital, Ningbo, Zheijiang 315000, P.R. China
}

Received August 19, 2019; Accepted March 17, 2020

DOI: $10.3892 / \mathrm{etm} .2020 .8758$

\begin{abstract}
Parkinson's disease (PD) is a chronic progressive disease that affects the central nervous system with a variety of symptoms. Although the precise etiology of PD is not yet fully understood, there is evidence to suggest that $\mathrm{T}$ cells serve an important role in the pathogenesis of PD. However, how $\mathrm{T}$ cells are recruited in the brain tissue remains to be elucidated. The present study utilized human samples from patients with and without PD to investigate the infiltration of $\mathrm{T}$ cells in lesions in the central nervous system. A chemically-induced mouse PD model was also used to investigate the roles of $\mathrm{T}$ cells in the pathogenesis of PD. Depletion of $\mathrm{CD}^{+}{ }^{+}$or $\mathrm{CD}^{+} \mathrm{T}$ cells was achieved using neutralizing antibodies. Adhesion molecule levels were assessed by flow cytometry. The results of the study indicated that $\mathrm{T}$ cell infiltration was evident in both human and murine samples of PD. Blocking $\mathrm{CD}^{+}$or $\mathrm{CD} 8^{+} \mathrm{T}$ cells attenuated the severity of murine PD. Intercellular adhesion molecule 1 (ICAM1 or CD54) was upregulated in mouse PD compared with controls, and its receptor, lymphocyte function-associated antigen-1 (LFA1) was overexpressed in $\mathrm{T}$ cells of the brain in PD mice compared with controls. Furthermore, inhibition of ICAM1 or LFA1 attenuated PD-associated characteristics in mice. In conclusion, the interaction between ICAM1 and LFA1 plays a role in
\end{abstract}

Correspondence to: Dr Jianqing Wu, Department of Geriatrics, Jiangsu Provincial Key Laboratory of Geriatrics, First Affiliated Hospital of Nanjing Medical University, 300 Guangzhou Road, Nanjing, Jiangsu 210000, P.R. China

E-mail: njwujianqing@163.com

Dr Qi Yao, Department of Geriatrics, Ningbo First Hospital, 59 Liuting Street, Ningbo, Zheijiang 315000, P.R. China

E-mail: nbyaoqi@sina.com

${ }^{*}$ Contributed equally

Key words: Parkinson's disease, central nervous system, inflammation, adhesion molecules, $\mathrm{T}$ cells recruiting $\mathrm{T}$ cells to the central nervous system to mediate experimental PD.

\section{Introduction}

Parkinson's disease (PD) is a common, progressive degenerative disease of the central nervous system that causes loss of steady motor function (1). PD is clinically characterized by resting tremor, bradykinesia, rigidity, impairment of balance, akinesia, postural reflex loss and other non-motor symptoms (1-4). The pathophysiological changes of PD are associated with the death of dopaminergic neurons in the substantia nigra pars compacta ( $\mathrm{SNpc}$ ), which subsequently leads to a lack of dopamine production and/or release in the striatum and motor impairment (5).

In addition to traditional pathological findings, such as intracellular inclusions containing $\alpha$-synuclein during post-mortem diagnosis $(6,7)$, there is evidence to indicate that PD is an inflammatory disease. Microglia are activated and express human leukocyte antigen DR isotype molecules in patients with PD (8). T-cell infiltration, which is absent in normal brain tissue, has been observed in samples from patients with PD $(9,10)$, indicating that $\mathrm{T}$ cells might play a role during the inflammatory pathogenesis of PD.

Inflammation observed in patients with PD has raised questions, such as how $\mathrm{T}$ cells migrate through the blood-brain barrier, and whether blocking T-cell infiltration can attenuate and slow the progression of PD $(11,12)$. Cellular adhesion molecules such as intercellular adhesion molecule 1 (ICAM1 or CD54) are upregulated during inflammation in the central nervous system and play an important role in T-cell recruitment $(11,12)$. However, the involvement of ICAM1 in the pathogenesis of PD remains to be elucidated. The present study investigated whether ICAM1 and its ligand lymphocyte function-associated antigen 1 (LFA-1) interact with each other to mediate T-cell infiltration. The results revealed that T-cell infiltration was evident in patients with PD and mice with experimental $\mathrm{PD}$, depletion of $\mathrm{CD}^{+} \mathrm{T}$ cells or $\mathrm{CD}^{+} \mathrm{T}$ cells attenuated the severity of experimental PD, ICAM1 was upregulated in the brain tissue of experimental PD mice, and blocking the ICAM1-LFA1 axis reduced T-cell infiltration and the severity of PD in experimental mice. 


\section{Materials and methods}

Ethics consideration. The ethics committee of Ningbo First Hospital approved and supervised the proposed experimental protocol (approval no: 2017-R044). Written consent forms were explained, agreed to and signed by the next-of-kin of deceased patients with PD and patients without central nervous system disease.

Histological diagnosis of $P D$. Brain tissues were obtained from post-mortem individuals (PD patients, 4 males and 4 females; age range, 67-89 years) admitted to Ningbo First Hospital between January and June 2017. Sex- and age-matched patients without PD served as controls. Brain tissues were processed as frozen sections. Serial sections (4-6 $\mu \mathrm{m})$ were cut. Slides were fixed with $10 \%$ formalin for $30 \mathrm{~min}$ at room temperature (RT), and stained with hematoxylin and eosin (Sigma-Aldrich; Merck KGaA) according to the manufacturer's instructions. PD diagnosis was confirmed for patients with PD or excluded for control patients in line with a published criterion by two experienced pathologists (13).

Immunofluorescence staining. Brain tissue obtained from the $\mathrm{SNpc}$ of human or mouse samples was processed as frozen sections and cut into serial sections of thickness 4-6 $\mu \mathrm{m}$. Slides were fixed in $10 \%$ formalin for $30 \mathrm{~min}$ at RT and blocked with $10 \%$ goat serum (Gibco; Thermo Fisher Scientific, Inc.) for $30 \mathrm{~min}$ at RT. Samples were then incubated with fluorescence-conjugated antibodies for $30 \mathrm{~min}$ at RT, which are listed in Table I. The number of tyrosine hydroxylase (TH)-producing cells in the lesions of the brain was used as an indicator for disease severity as previously reported (10). Briefly, histological sections were stained with anti-TH primary antibodies for $30 \mathrm{~min}$ at RT (Table I). After washing with PBS, slices were stained with anti-rabbit AF647-conjugated secondary antibodies (1:1,000, Thermo Fisher Scientific, Inc.; cat. no. A32733) for $30 \mathrm{~min}$ at RT.

Experimental PD induction. A total of 395 Male C57/B16 mice, aged 10-12 weeks old, were purchased from the Jackson Laboratory. Mice were kept in a specific pathogen-free environment with controlled temperature $\left(23^{\circ} \mathrm{C}\right)$ and humidity (40-60\%), under a 12 -h light/dark cycle. Mice were allowed ad libitum access to food and water. Experimental PD was induced by intraperitoneally injecting 1-methyl-4-phenyl-1,2,3,6-tetrahydropyridine (MPTP; Sigma-Aldrich; Merck KGaA) according to a published protocol (10). Briefly, MPTP $(20 \mathrm{mg} / \mathrm{kg})$ in $100 \mathrm{ml}$ of saline was intraperitoneally injected 4 times at 2 -h intervals, and mice were sacrificed by cervical dislocation under general anesthesia by isoflurane inhalation (oxygen flow, $0.81 / \mathrm{min}$; isoflurane vaporizer, $3 \%$ ) at designated time points (4 days after MPTP injection) following the last injection. Control mice received $100 \mathrm{ml}$ saline via intraperitoneal injection. A total of 9 mice per group were utilized in each experiment, which was repeated in triplicate.

Open field tests. Behavioral assessments were performed 4 days following MPTP injection using open field tests, which are the most common tests for behavior of MPTP-treated PD mice (14). The experimental procedure was adapted from a previous publication (15). All tests were performed between 12:00 and 2:00 p.m. in normal lighting. Briefly, a white plastic rectangular box with dimensions of $80 \times 40 \times 20 \mathrm{~cm}$ was utilized for open field tests. The bottom of the box was drawn with a grid of $5 \times 5-\mathrm{cm}^{2}$ squares. The total distance that a mouse moved was manually measured by counting the number of squares. Rearing, which represents exploratory activity, and grooming, which indicates displacement response, were scored when a mouse was placed in the open field for 5 min (16).

Neutralizing antibodies. The abundance of $\mathrm{CD} 8^{+}$and $\mathrm{CD} 4^{+}$ $\mathrm{T}$ cells as well as the efficacy of deletion of these two cell types in the brain tissue were determined by immunofluorescence. Circulating $\mathrm{CD} 8^{+}$and $\mathrm{CD} 4^{+} \mathrm{T}$ cells were also measured by flow cytometry when neutralizing antibodies were applied. To deplete $\mathrm{CD} 8{ }^{+} \mathrm{T}$ cells, an anti-CD8 antibody (clone 2.43; Bio X cell; cat. no. BP0061) at a dose of $250 \mu \mathrm{g}$ in $100 \mu \mathrm{l}$ PBS was intraperitoneally injected at days -7 and -3 before MPTP injection (day 0). Control mice received isotype control antibodies (rat anti-mouse IgG2b; Bio X Cell; cat. no. BP0090) following the same regimen as $\mathrm{CD} 8^{+} \mathrm{T}$ cell depletion.

To deplete $\mathrm{CD} 4{ }^{+} \mathrm{T}$ cells, anti-CD4 antibodies (cloneGK1.5; Bio X Cell; cat. no. BP0003-1) were administered at a dose of $250 \mu \mathrm{g}$ in $100 \mu \mathrm{l}$ PBS by intraperitoneal injection at days-7 and -3. Control mice received isotype control antibodies (rat anti-mouse IgG2b; Bio X Cell; cat. no. BP0090) using the same regimen of anti-CD4 antibodies.

Cell type-specific expression of ICAM-1 and LFA1 was determined by flow cytometry. For blocking of ICAM1, anti-ICAM1 antibodies (clone YN1/1.7.4; Thermo Fisher Scientific, Inc.) were administered at a dose of $4 \mathrm{mg} / \mathrm{kg}$ by intraperitoneal injection at days-7 and -3. Control mice received

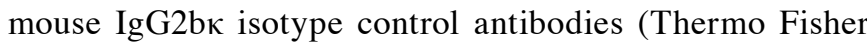
Scientific,Inc.) following the same regimen as ICAM-1 blockade. For blocking of LFA1, anti-CD11a antibodies (clone M17/4; Thermo Fisher Scientific, Inc.) were intraperitoneally injected at a dose of $100 \mu \mathrm{g}$ at days-7 and -3. Control mice received mouse $\mathrm{IgG} 2 \mathrm{~b} \kappa$ isotype control antibodies following the same regimen as LFA-lblockade. A total of 9 mice per group were utilized in each experiment, which was repeated in triplicate.

Flow cytometry. Mouse brain tissue was processed using a commercial kit for brain tissue dissociation (Miltenyi Biotec, Inc.; cat. no. 130-107-677) to obtain single-cell suspensions according to the manufacturer's instructions. Circulating T cells were obtained by lysing red blood cells for $5 \mathrm{~min}$ at RT and centrifugation at $300 \mathrm{x}$ g for $10 \mathrm{~min}$ at RT. Splenic T cells were obtained by mechanical isolation using a cell strainer and centrifugation at $300 \mathrm{xg}$ for $10 \mathrm{~min}$ at RT.

Cells were treated with anti-CD16/32 antibody (1:200; Thermo Fisher Scientific, Inc.; cat. no. 14-0161) for $10 \mathrm{~min}$ at RT, and then stained with the designated antibodies listed in Table I. Following incubation with the corresponding antibodies, cells were analyzed with an Attune NxT flow cytometer (Thermo Fisher Scientific, Inc.). Data were presented and analyzed using Kaluza 1.3 (Beckman Coulter, Inc.).

Statistical analysis. Data are presented as the mean \pm SD. Data were compared using Student's t-test for two groups or 
Table I. Antibodies used for immunostaining and flow cytometry.

\begin{tabular}{|c|c|c|c|}
\hline Antibody (clone) & Manufacturer (cat. no.) & Utilization & Dilution \\
\hline Tyrosine hydroxylase $(\mathrm{TH})$ & Abcam (ab112) & IF & $1: 1,000$ \\
\hline CD8-PE (53-6.7) & eBioscience; Thermo Fisher Scientific, Inc. (12-0081-82) & IF, FC & $1: 100$ \\
\hline CD4-PE (RM4-5) & eBioscience; Thermo Fisher Scientific, Inc. (12-0042-82) & IF, FC & $1: 100$ \\
\hline CD45-APC (30-F11) & eBioscience; Thermo Fisher Scientific, Inc. (17-0451-82) & $\mathrm{FC}$ & $1: 100$ \\
\hline CD31-PE (390) & eBioscience; Thermo Fisher Scientific, Inc. (12-0311-82) & FC & $1: 100$ \\
\hline ICAM1-FITC (YN1/1.7.4) & eBioscience; Thermo Fisher Scientific, Inc. (11-0541-82) & $\mathrm{FC}$ & $1: 100$ \\
\hline CD3-PE (145-2C11) & eBioscience; Thermo Fisher Scientific, Inc. (12-0031-82) & $\mathrm{FC}$ & $1: 100$ \\
\hline LFA1-FITC (M18/2) & eBioscience; Thermo Fisher Scientific, Inc. (11-0181-82) & $\mathrm{FC}$ & $1: 100$ \\
\hline
\end{tabular}

PE, phycoerythrin; APC, allophycocyanin; ICAM1, intercellular adhesion molecule 1; LFA1, lymphocyte function-associated antigen-1; IF, immunofluorescence; FC, flow cytometry.

one-way ANOVA with Bonferroni's post hoc test for multiple groups. Associations between the abundance of $\mathrm{CD}^{+}$or $\mathrm{CD}^{+} \mathrm{T}$ cells and severity of PD were analyzed using linear regression. $\mathrm{P}<0.05$ was considered to indicate a statistically significant difference. Statistical analysis was performed using GraphPad Prism 7 (GraphPad Software, Inc.).

\section{Results}

T-cell infiltration is observed in the brain tissue of PD patients and MPTP-intoxicated mice. To explore T-cell infiltration in the brain tissue of patients with PD, post-mortem samples were used for immunofluorescence staining. In agreement with previous reports $(10,17), \mathrm{CD}^{+} \mathrm{T}$ cells and $\mathrm{CD}^{+} \mathrm{T}$ cells were observed in the brain tissue of patients with PD, but were expressed at a significantly lower level in patients without PD (Fig. 1A and B). CD8 ${ }^{+}$T-cell infiltration positively associated with disease duration (Fig. 1C), suggesting that $\mathrm{CD}^{+} \mathrm{T}$-cell infiltration might contribute to disease severity. Subsequently, the MPTP-intoxicated mouse model of PD was used to study T-cell recruitment in an experimental setting. Mice treated with MPTP gradually manifested symptoms of hypoactivity beginning at $12 \mathrm{~h}$ after MPTP injection. Four days after MPTP injection, mice treated with MPTP also harbored $\mathrm{CD}^{+}$and $\mathrm{CD}^{+} \mathrm{T}$ cells in the brain tissue, mainly in the SNpc and striatum regions (Figs. 1D and E; S1); whereas these two types of immune cells were not evident in saline-treated mice. These results indicate that T-cell infiltration is a feature in patients with PD and experimental PD mice.

Depletion of $C D 4^{+} T$ cells or $C D 8^{+} T$ cells attenuates experimental $P D$. Next, it was determined whether the disease severity in the mouse model of PD was $\mathrm{CD}^{+} \mathrm{T}$ cell- or $\mathrm{CD} 8^{+}$ $\mathrm{T}$ cell-dependent. Neutralizing antibodies or matched isotype control antibodies were used to deplete $\mathrm{CD}^{+} \mathrm{T}$ cells or $\mathrm{CD}^{+}$ $\mathrm{T}$ cells in MPTP-treated mice. The efficacy of $\mathrm{CD}^{+}$or $\mathrm{CD}^{+}$ T-cell depletion was determined by measuring circulating $\mathrm{T}$ cells using flow cytometry (data not shown). Depletion of $\mathrm{CD}^{+}{ }^{+} \mathrm{T}$ cells and $\mathrm{CD}^{+} \mathrm{T}$ cells in the lesions of MPTP-treated mice was also confirmed by immunofluorescence (Fig. 2A). As shown in Fig. 2B and C, depletion of $\mathrm{CD}^{+} \mathrm{T}$ cells attenuated disease severity, as indicated by the increased number of $\mathrm{TH}^{+}$ cells at 4 days after MPTP injection compared with isotype control antibody-treated mice. The behaviour of the mice was also observed using open field tests. As shown in Fig. 2D-F, depletion of $\mathrm{CD}^{+}$attenuated behavioral dysfunctions in MPTP-treated mice compared with isotype control antibody-treated mice. Similar results were also observed in mice treated with anti-CD8 neutralizing antibodies (Fig. 2C-F). These data suggest that $\mathrm{CD}^{+}$and $\mathrm{CD}^{+} \mathrm{T}$ cells play a detrimental role in the pathogenesis of experimental PD.

ICAM1 expression in endothelial cells and LFA1 expression in T cells are upregulated in MPTP-treated mice. Cellular adhesion molecules on endothelial cells play a role in the initiation of leukocyte infiltration through the blood-brain barrier (18). Thus, the expression of cellular adhesion molecules was investigated. According to the literature, ICAM1 is critical for T-cell recruitment in inflammatory brain conditions; LFA1, a major ligand of ICAM1, expressed by T cells interacts with ICAM1 to mediate T-cell infiltration in the brain during a number of conditions $(11,12)$. Therefore, the expression of ICAM1 in endothelial cells and LFA1 in T cells were investigated. Flow cytometry results showed that endothelial cells (defined as CD45-CD $31^{+}$cells) collected from the brain of MPTP-treated mice expressed high levels of ICAM1 (Fig. 3A and B) compared with saline-treated mice at day 4 after MPTP injection. Since saline-treated mice presented with very low T-cell infiltration in the brain, $\mathrm{T}$ cells collected from the brain, blood and spleen of MPTP-treated mice were analyzed. As shown in Fig. 3C and D, T cells collected from the brain expressed increased levels of LFA1 compared with circulating and splenic $\mathrm{T}$ cells, indicating that upregulation of LFA1 on T cells was specific to brain inflammation at day 4 after MPTP injection. These results indicate that the interaction between endothelial ICAM1 and LFA1 expressed by T cells might be involved in the process of MPTP-induced PD.

Blocking ICAMI or LFAl alleviates MPTP-induced PD in mice. Whether blocking the ICAM1/LFA1 axis could be used as a therapeutic strategy for experimental PD was investigated. Antibodies neutralizing ICAM1 or CD11a (a subunit of LFA1) 

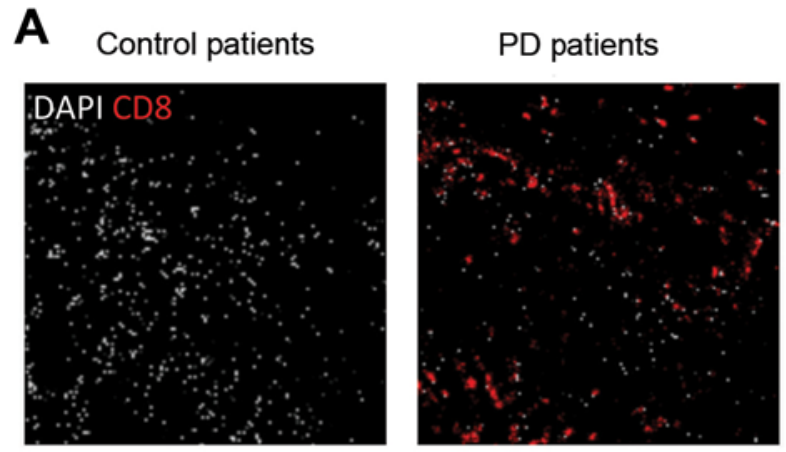

B
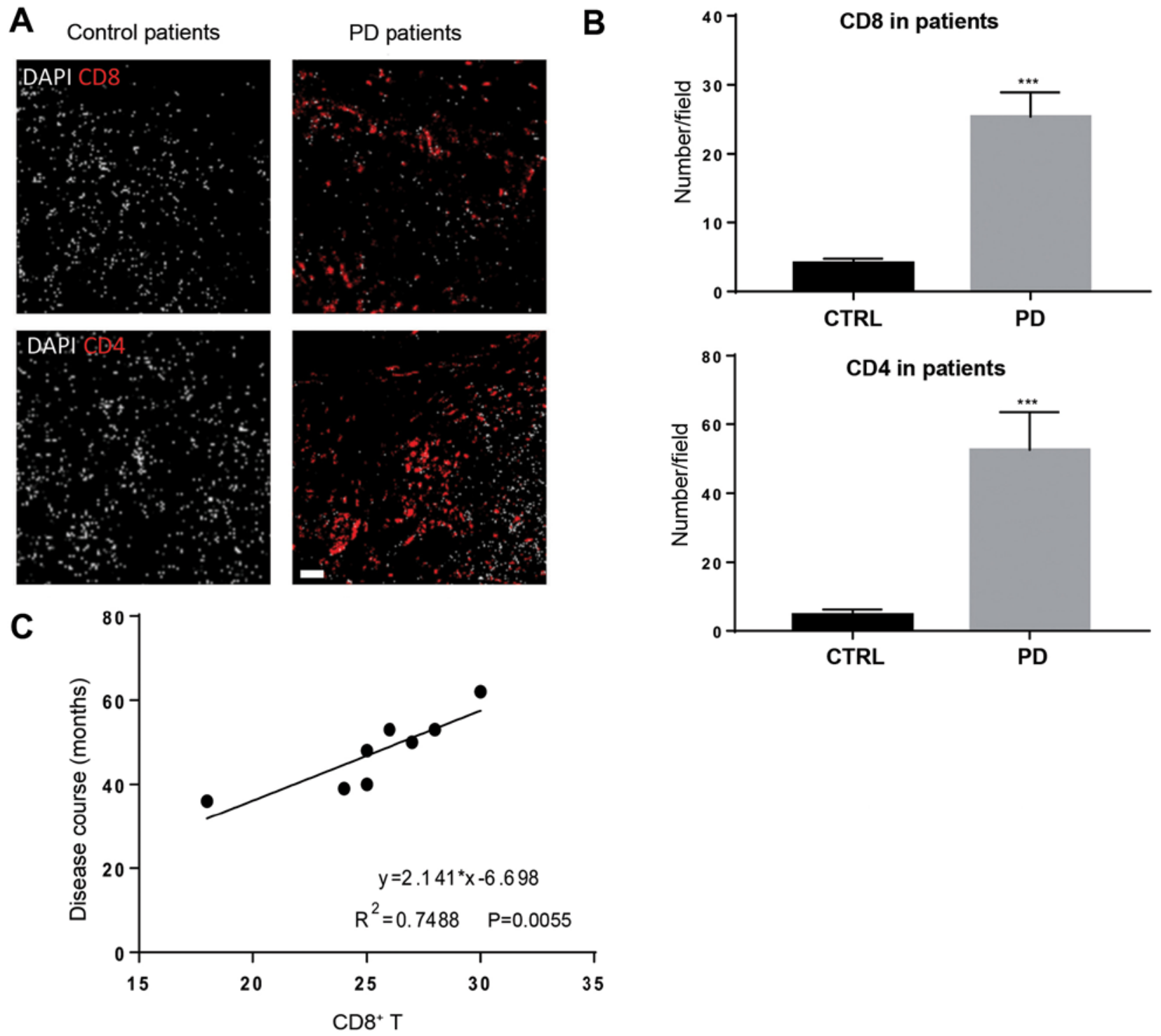

D

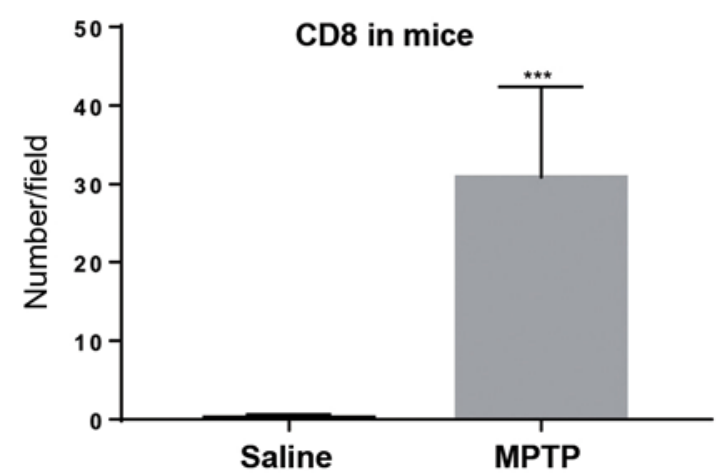

E

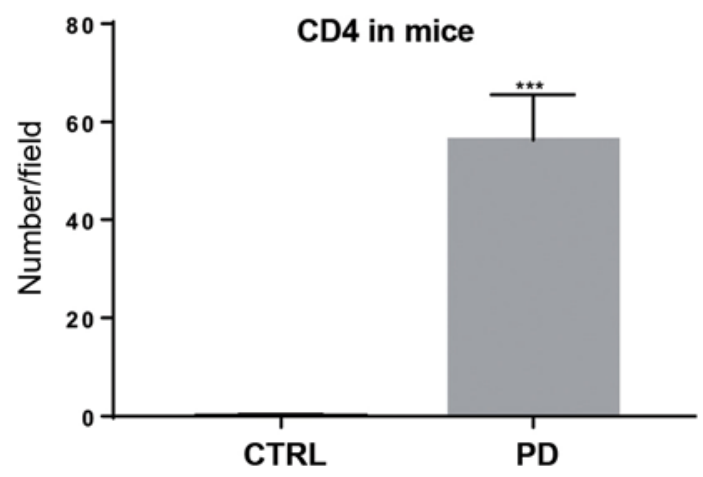

Figure 1. T-cell infiltration is observed in patients with PD and MPTP-treated mice. (A) CD8 ${ }^{+}$and $\mathrm{CD} 4^{+} \mathrm{T}$ cells were measured in patients with or without PD using immunofluorescence. Scale bars, $20 \mu \mathrm{m}$. (B) $\mathrm{CD}^{+}$and $\mathrm{CD} 4^{+} \mathrm{T}$-cell levels increased in the brain tissue of patients with PD compared with the control group. $n=8$. ${ }^{* * *} \mathrm{P}<0.001$. (C) The abundance of $\mathrm{CD} 8^{+} \mathrm{T}$ cells in the brain tissue positively associated with disease duration. MPTP-treated mice also harbored increased levels of (D) $\mathrm{CD}^{+}$and (E) $\mathrm{CD} 4^{+} \mathrm{T}$ cells in the brain tissue 4 days after MPTP injection. $\mathrm{n}=9$. Experiments were performed in triplicate. ${ }^{* * *} \mathrm{P}<0.001$. PD, Parkinson's disease; MPTP, 1-methyl-4-phenyl-1,2,3,6-tetrahydropyridine; CTRL, control.

were administered to MPTP-treated mice, while matched isotype control antibodies were used as controls. Blocking ICAM1 or its ligand LFA1 significantly reduced disease severity in MPTP-challenged mice as measured by the number of $\mathrm{TH}^{+}$cells (Figs. 4A; S2) and behavior (Fig. 4B-D) compared with isotype antibody-administered mice. Subsequently, anti-ICAM1 and anti-CD11a antibodies were co-administered to experimental animals; no synergistic effects were observed 
A

CD4 depletion

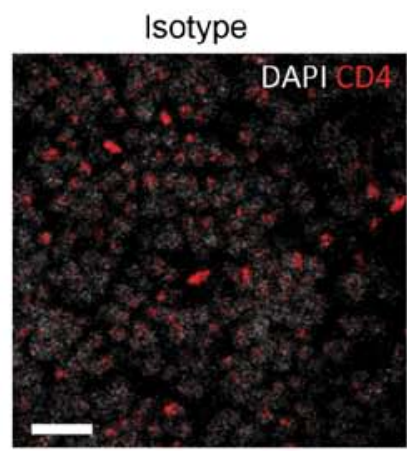

B

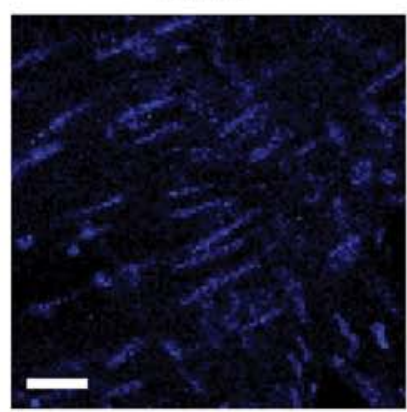

C

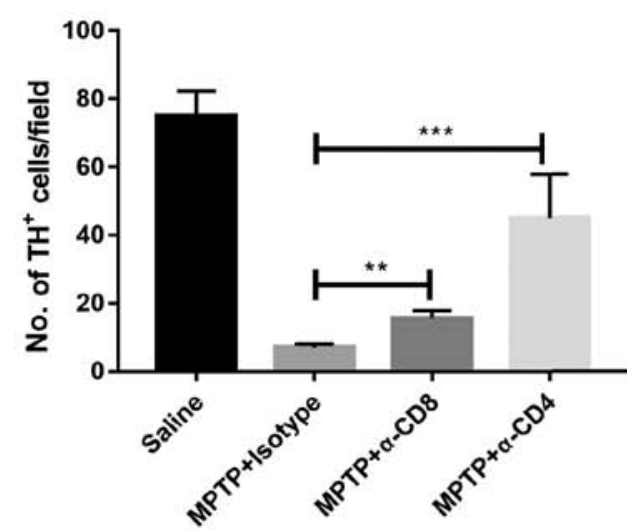

Treatment

E

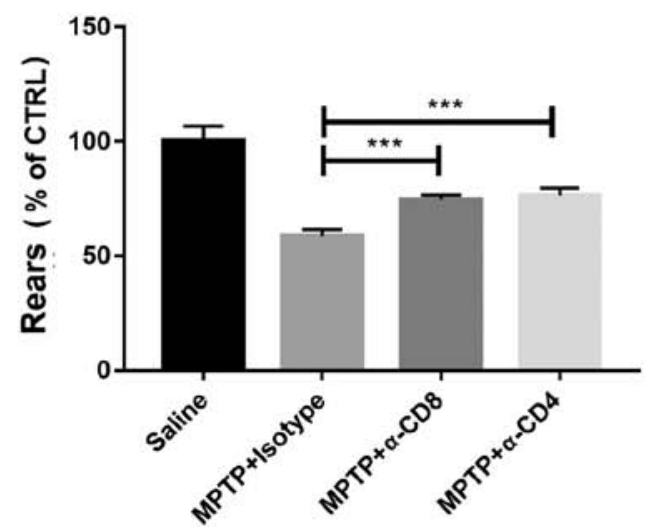

Treatment
CD8 depletion
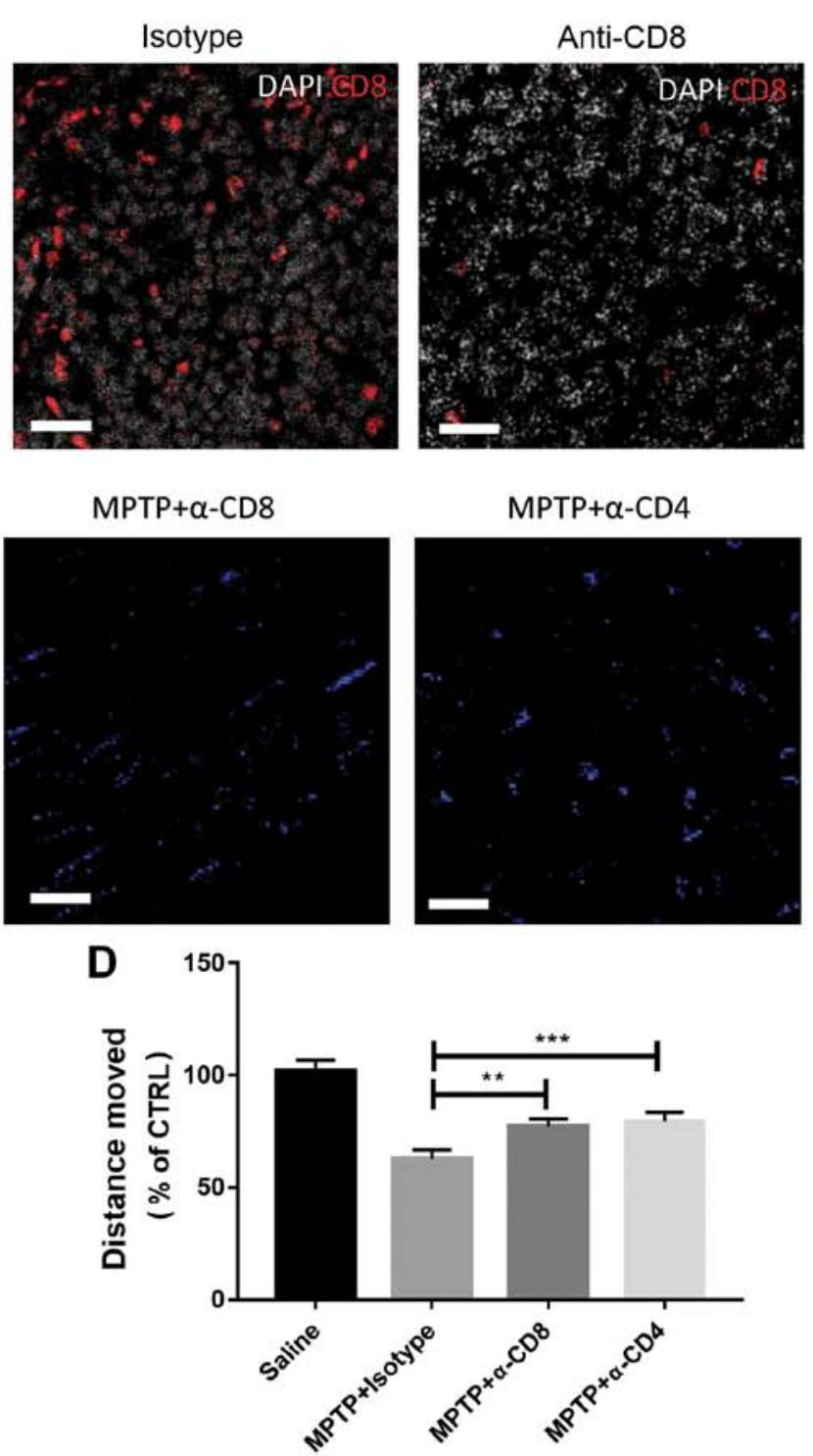

Treatment

$\mathbf{F}$

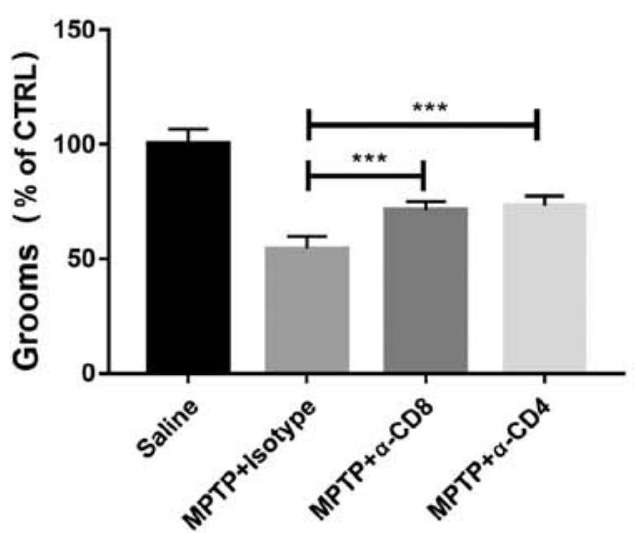

Treatment

Figure 2. Depletion of $\mathrm{CD}^{+}$or $\mathrm{CD}^{+} \mathrm{T}$ cells attenuates MPTP-induced brain damage. (A) Depletion of $\mathrm{CD}^{+}$and $\mathrm{CD} 8^{+} \mathrm{T}$ cells in MPTP-treated mice was confirmed by immunofluorescence. Scale bars, $20 \mu \mathrm{m}$. (B) Representative images of $\mathrm{TH}^{+}$cell staining (visualized by AF-647 secondary antibodies, blue in the images) in the brain tissue of mice. Scale bars, $20 \mu \mathrm{m}$. (C) Depletion of CD8 ${ }^{+}$or CD4 ${ }^{+}$T cells attenuated the severity of MPTP-induced Parkinson's disease in mice as measured by the abundance of $\mathrm{TH}^{+}$cells. Mouse behavior, including (D) distance moved, (E) rears and (F) grooms were assessed by open field tests. MPTP-treated mice showed impaired behavior, but depletion of CD4 ${ }^{+}$or $\mathrm{CD}^{+} \mathrm{T}$ cells attenuated behavioral dysfunction. $\mathrm{n}=9$. Experiments were performed in triplicate. ${ }^{* *} \mathrm{P}<0.01$ and ${ }^{* * *} \mathrm{P}<0.001$. MPTP, 1-methyl-4-phenyl-1,2,3,6-tetrahydropyridine; CTRL, control; TH, tyrosine hydroxylase; $\alpha$-CD4, anti-CD4 antibodies; $\alpha$-CD8, anti-CD8 antibodies. 
A

$$
\text { Gated on } \mathrm{CD}^{-} 5^{-} \mathrm{CD} 31^{+}
$$

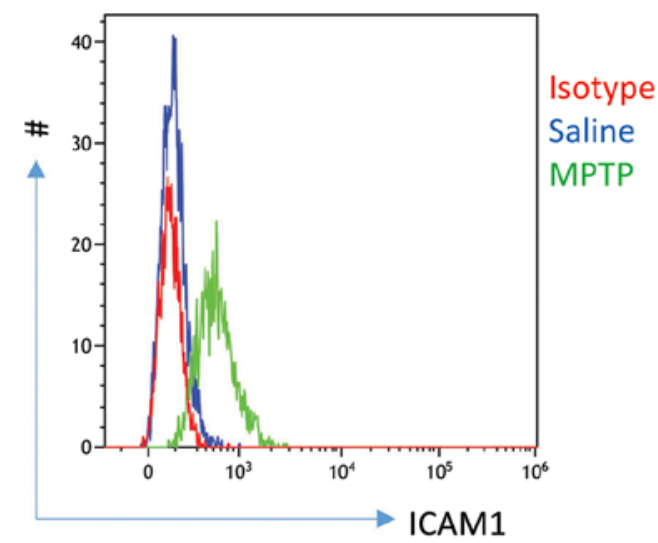

C

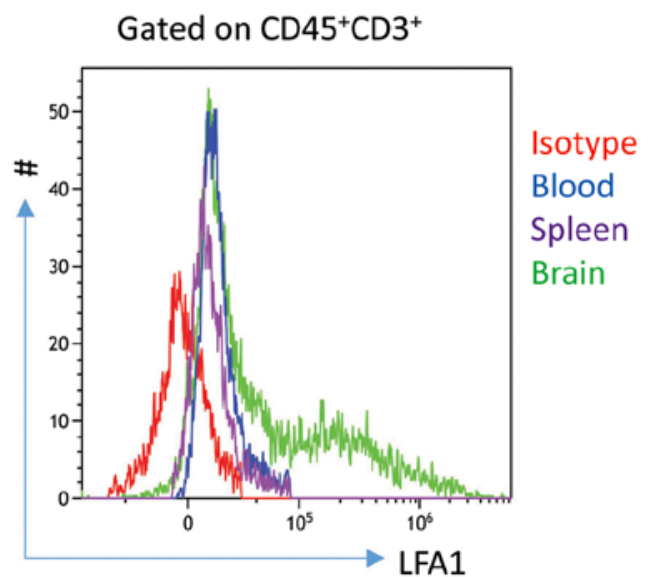

B

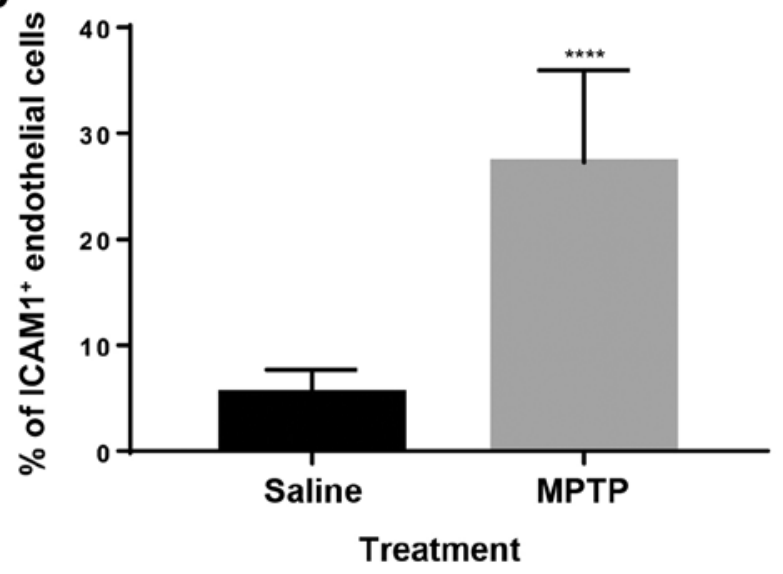

D

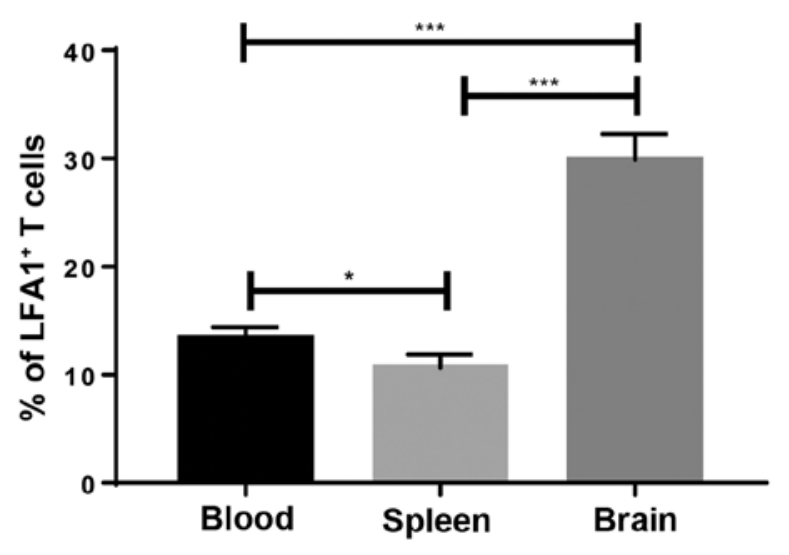

Figure 3. ICAM1 is upregulated in brain endothelial cells and LFA1 is elevated in brain tissue T cells collected from MPTP-treated mice. (A) Representative images of ICAM1 in brain tissue endothelial cells of saline- or MPTP- treated mice. MPTP-treated mice exhibited increased levels of ICAM1. (B) Endothelial cells collected from the brains of MPTP-treated mice expressed significantly higher levels of ICAM1 4 days after MPTP injection ( $\mathrm{n}=9$ ). Experiments were performed in triplicate. ${ }^{* * * *} \mathrm{P}<0.0001$. (C) Representative images of LFA1 expressed in different tissues of MPTP-treated mice. Brain tissue exhibited higher expressions of LFA1 on T cells. T cells $\left(\mathrm{CD} 45^{+} \mathrm{CD}^{+}\right)$were collected from the blood, spleen and brain of MPTP-treated mice. (D) T cells in the brain of MPTP-treated mice expressed significantly increased levels of LFA1 compared with the blood and spleen at day 4 after MPTP injection. n=9. Experiments were performed in triplicate. ${ }^{*} \mathrm{P}<0.05$ and ${ }^{* * * *} \mathrm{P}<0.001$. ICAM1, intercellular adhesion molecule 1 ; LFA1, lymphocyte function-associated antigen-1; MPTP, 1-methyl-4-phenyl-1,2,3,6-tetrahydropyridine.

(data not shown), indicating the possibility that the interaction between ICAM1 and LFA1 in this setting might be specific with no involvement of any other receptors or ligands. Therefore, blocking ICAM1 or LFA1 may have therapeutic potential in PD.

\section{Discussion}

The present study demonstrated that $\mathrm{T}$ cells play a role during the pathogenesis of $\mathrm{PD}$. The results indicate that T-cell infiltration is a universal feature in patients with PD and experimental PD mice. Depletion of $\mathrm{CD}^{+}$or $\mathrm{CD}^{+} \mathrm{T}$ cells by the administration of neutralizing antibodies attenuated the severity of experimental PD in mice as assessed by the abundance of $\mathrm{TH}^{+}$cells in the brain tissue and behavioral assessment by open field tests. Moreover, endothelial ICAM1 and LFA1 expressed on T cells appear to be involved during the process of T cell recruitment, as inhibiting T-cell recruitment by blocking the ICAM1-LFA1 axis alleviated experimental PD. Therefore, the present study highlighted the therapeutic potential of using strategies where the ICAM1-LFA1 axis is inhibited for treatment of PD.

$\mathrm{PD}$ is a progressive degenerative disease of the central nervous system that affects millions of individuals worldwide (1-4). The precise etiology of PD has not yet been fully elucidated, where the loss of dopamine-producing neurons in the SNpc mediates the pathogenesis (10). A growing body of evidence, including findings in the present study, indicate that aberrant immune responses in the brain contribute to the pathogenesis of PD (1-4,10). It has been reported that $\mathrm{T}$ cells are recruited into the SNpc during experimental PD $(9,10)$. T-cell infiltration was also observed in the brain tissue of patients with PD and mice with experimental PD in the present study. Considering that dysregulated inflammation in the brain occurs in other central nervous system diseases such as Alzheimer's disease and multiple sclerosis (1-4), more research should be focused on the immunopathogenesis of these brain 
A
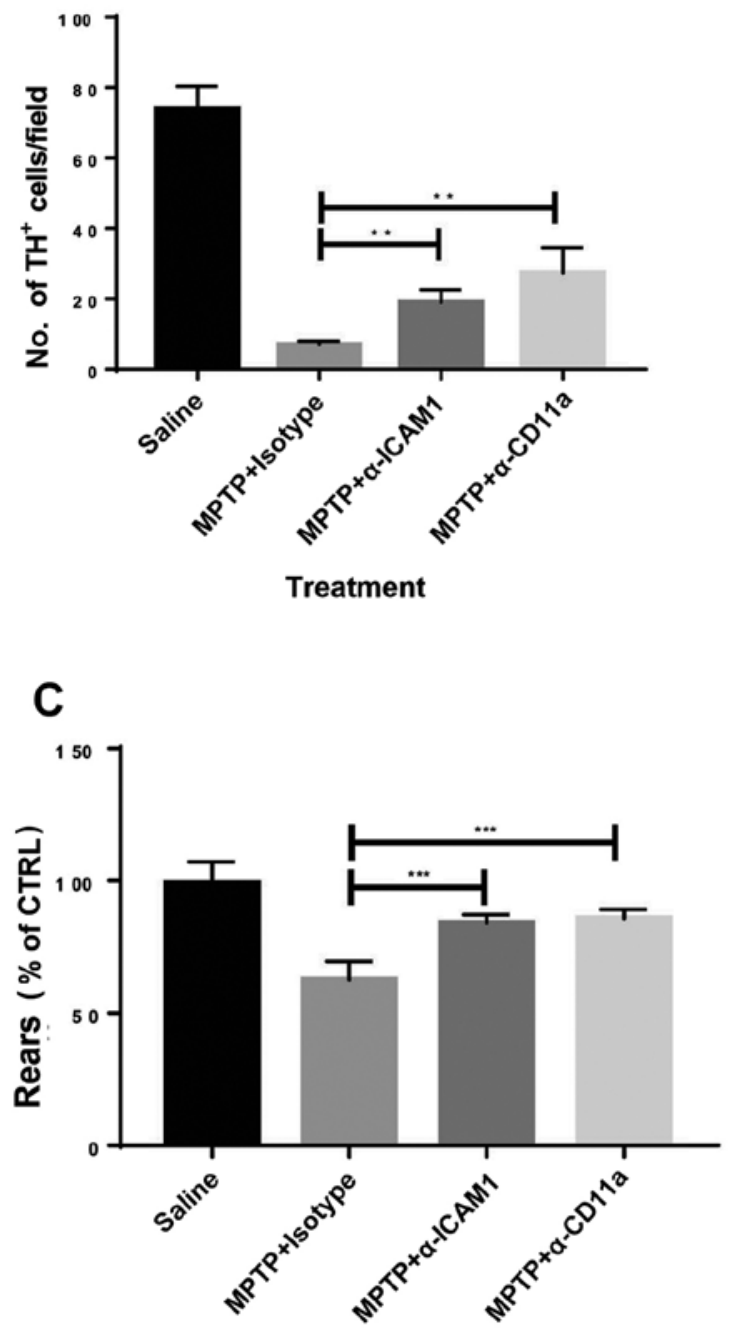

Treatment
B

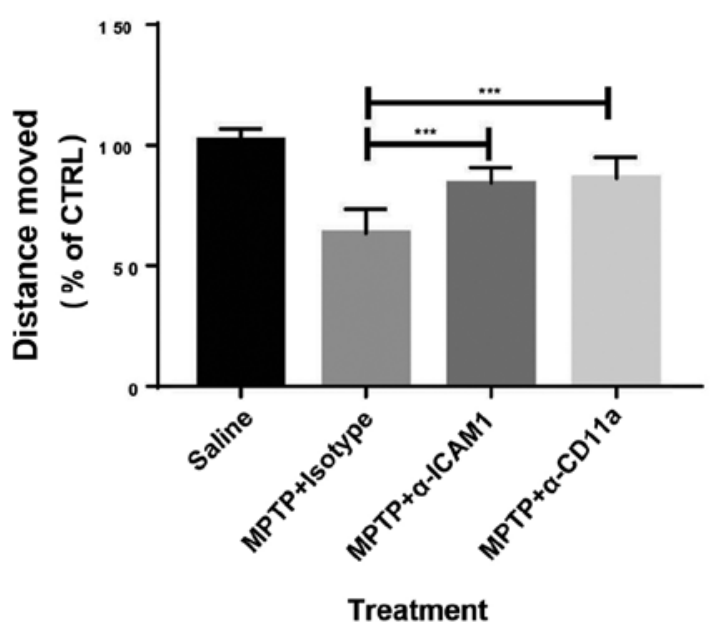

D

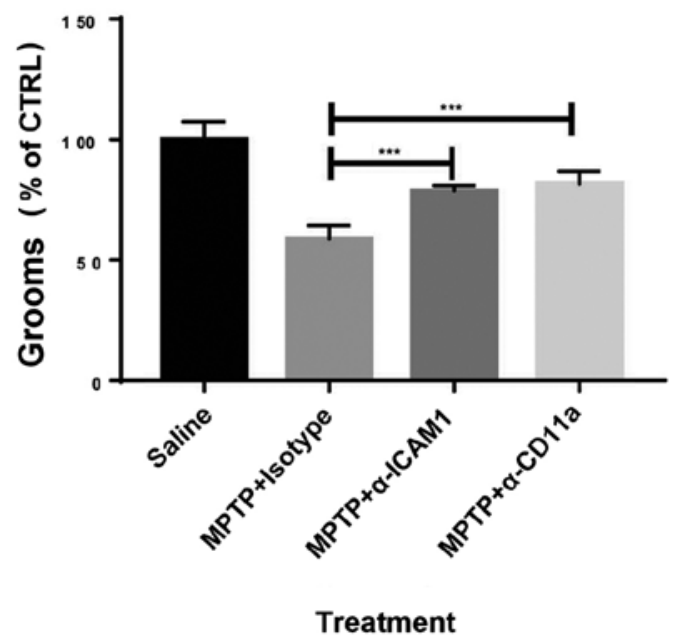

Figure 4. Blocking of ICAM1 or CD11a attenuates the severity of MPTP-induced PD in mice. (A) Blocking of ICAM1 or CD11a increased the levels of TH ${ }^{+}$ cells at day 4 after MPTP injection. Mouse behaviour assessed by open field tests showed improved clinical presentation in those treated with ICAM1 or CD11a blockade at day 4 after MPTP injection, including (B) distance moved, (C) rears and (D) grooms. $n=9$. Experiments were performed in triplicate. ${ }^{* *} \mathrm{P}<0.01$ and ${ }^{* * *} \mathrm{P}<0.001$. ICAM1, intercellular adhesion molecule 1; MPTP, 1-methyl-4-phenyl-1,2,3,6-tetrahydropyridine; TH, tyrosine hydroxylase; CTRL, control; $\alpha$-ICAM1, anti-ICAM1 antibodies; $\alpha$-CD11a, anti-CD11a antibodies.

conditions and how to manipulate the aberrant inflammatory responses in order to treat these diseases.

Interactions between ICAM1 and LFA1 are critical during the recruitment of leukocytes to inflamed sites (19). The ICAM1-LFA1 axis is overactivated in patients with PD (20). Although ICAM1 or LFA1 expression was not assessed in the human cohort of the present study, it is possible that PD patients in the present cohort have high expressions of ICAM1 and LFA1, although this warrants further examination. The present study found that endothelial ICAM1 is upregulated in mice with experimental PD compared with control mice. The role of the ICAM1-LFA1 axis in PD was also investigated. Notably, the aforementioned study (20) did not conduct functional assays in terms of disease severity when the axis is inhibited, whereas the present data indicated the roles of the ICAM1-LFA1 axis in the pathogenesis of PD. T cells in the SNpc of mice treated with MPTP expressed increased levels of LFA1 compared with T cells harvested from the peripheral circulation or the spleen, indicating that $\mathrm{T}$ cells in the brain tissue of mice with PD are more activated. In the process of experimental PD, endothelial cells in the brain tissue harbor increased ICAM1 expression. Circulating T cells in the inflamed area of the brain upregulate the expression of LFA1 to interact with ICAM1 expressed by endothelial cells. This interaction further activates $\mathrm{T}$ cells to begin the multiple-step process of T-cell recruitment (21), including capture, rolling, adhesion and transmigration, which involves the interaction between ICAM1 and LFA1. However, the present study did not investigate in which of the aforementioned steps the interaction between ICAM1 and LFA1 plays the most prominent effect. Advanced imaging techniques such intravital microscopy will provide a means to trace each step of leukocyte recruitment in order to elucidate the precise influence of the ICAM1-LFA1 axis on PD pathogenesis.

T-cell infiltration is a hallmark of PD in humans and experimental PD in mice (10). Depletion of $\mathrm{CD}^{+}$or $\mathrm{CD}^{+} \mathrm{T}$ cells 
resulted in improved disease status, indicating that $\mathrm{T}$ cells in the brain tissue are detrimental. The current study speculates that there might be several theories as to how $\mathrm{T}$ cells execute pathological effects in PD. First, $\mathrm{CD}^{+} \mathrm{T}$ cells exert cytotoxic effects in the setting of PD to mediate cell death in the inflamed brain tissue. Second, CD4 ${ }^{+} \mathrm{T}$ cells, possibly T helper (Th) 1 and Th17 cells, produce pro-inflammatory cytokines such as tumor necrosis factor (TNF)- $\alpha$ to increase apoptosis of neurons. Pathogenic Th17 cells expressing TNF- $\alpha$ and IL17 have been observed in other inflammatory conditions $(22,23)$, and might also contribute to the pathogenesis of PD. Chemokines secreted by $\mathrm{T}$ cells might recruit other leukocytes such monocytes and neutrophils to perform cell-killing effects. It is worth noting that more studies are warranted for the precise detrimental roles of $\mathrm{T}$ cells in the pathogenesis of PD.

A limitation of the present study is that the levels of $\mathrm{CD}^{+}$or $\mathrm{CD}^{+} \mathrm{T}$ cells in the central nervous system when the ICAM1-LFA1 axis is inhibited were not measured. However, the present study showed that when the ICAM1-LFA1 axis is inhibited or when $\mathrm{T}$ cells are depleted, the disease severity is attenuated. Hence, it is hypothesized that the abundance of $\mathrm{T}$ cells in the brain lesions would decrease when the ICAM1-LFA1 axis is inhibited. The roles of the axis in T-cell recruitment during the pathogenesis of PD are worthy of exploration.

Intervention in the ICAM1-LFA1 axis has possible therapeutic benefits for patients with PD. The depletion of $\mathrm{T}$ cells and also inhibition of T-cell recruitment attenuated the severity of PD, highlighting the potential of intervention in the ICAM1-LFA1 axis in a clinical setting. Blocking T-cell or other leukocyte recruitment has been shown to be effective in a number of inflammatory conditions, including inflammatory bowel disease and multiple sclerosis, and so it may also be a possible therapeutic strategy for patients with PD.

In conclusion, ICAM1 expressed in endothelial cells and LFA1 expressed in $\mathrm{T}$ cells play an essential role in $\mathrm{T}$ cell recruitment during experimental $\mathrm{PD}$ in mice.

\section{Acknowledgements}

Not applicable.

\section{Funding}

The current study was supported by Zhejiang Province Chinese Medicine Science Projects (grant no. 2018ZA108).

\section{Availability of data and materials}

The datasets used and/or analyzed during the current study are available from the corresponding author on reasonable request.

\section{Authors' contributions}

WL performed the majority of experiments and prepared the first draft of the manuscript. SC and YL performed histological, immunofluorescence and flow cytometry experiments. YX and QM performed statistical analysis. QY and JW conceptualized and supervised the current study. All authors read and approved the final manuscript.

\section{Ethics approval and consent to participate}

The ethics committee of Ningbo First Hospital approved and supervised the proposed experimental protocol (approval no: 2017-R044). Written informed consent was provided by the next-of-kin of the participants.

\section{Patient consent for publication}

Not applicable.

\section{Competing interests}

The authors declare that they have no competing interests.

\section{References}

1. Jankovic J: Parkinson's disease: Clinical features and diagnosis. J Neurol Neurosurg Psychiatry 79: 368-376, 2008.

2. Noorian AR, Rha J, Annerino DM, Bernhard D, Taylor GM and Greene JG: Alpha-synuclein transgenic mice display age-related slowing of gastrointestinal motility associated with transgene expression in the vagal system. Neurobiol Dis 48: 9-19, 2012.

3. Chaudhuri KR and Schapira AH: Non-motor symptoms of Parkinson's disease: Dopaminergic pathophysiology and treatment. Lancet Neurol 8: 464-474, 2009.

4. Magerkurth C, Schnitzer R and Braune S: Symptoms of autonomic failure in Parkinson's disease: Prevalence and impact on daily life. Clin Auton Res 15: 76-82, 2005.

5. Fasano $M$ and Lopiano L: Alpha-synuclein and Parkinson's disease: A proteomic view. Expert Rev Proteomics 5: 239-248, 2008.

6. Tofaris GK, Razzaq A, Ghetti B, Lilley KS and Spillantini MG: Ubiquitination of alpha-synuclein in Lewy bodies is a pathological event not associated with impairment of proteasome function. J Biol Chem 278: 44405-44411, 2003.

7. Spillantini MG, Schmidt ML, Lee VM, Trojanowski JQ, Jakes R and Goedert M: Alpha-synuclein in Lewy bodies. Nature 388: 839-880, 1997

8. Tansey MG, McCoy MK and Frank-Cannon TC: Neuroinflammatory mechanisms in Parkinson's disease: Potential environmental triggers, pathways, and targets for early therapeutic intervention. Exp Neurol 208: 1-25, 2007.

9. Saunders JA, Estes KA, Kosloski LM, Allen HE, Dempsey KM, Torres-Russotto DR, Meza JL, Santamaria PM, Bertoni JM, Murman DL, et al: CD4+ regulatory and effector/memory $\mathrm{T}$ cell subsets profile motor dysfunction in Parkinson's disease. J Neuroimmune Pharmacol 7: 927-938, 2012.

10. Brochard V, Combadière B, Prigent A, Laouar Y, Perrin A, Beray-Berthat V, Bonduelle O, Alvarez-Fischer D, Callebert J, Launay JM, et al: Infiltration of CD4+ lymphocytes into the brain contributes to neurodegeneration in a mouse model of Parkinson disease. J Clin Invest 119: 182-192, 2009.

11. Jiang L, Hu J, Feng J, Han D and Yang C: Substrate stiffness of endothelial cells directs LFA-1/ICAM-1 interaction: A physical trigger of immune-related diseases? Clin Hemorheol Microcirc 61: 633-643, 2016.

12. Harrer A, Pilz G, Wipfler P, Oppermann K, Sellner J, Hitzl W, Haschke-Becher E, Afazel S, Rispens T, van der Kleij D, et al: High interindividual variability in the CD4/CD8 T cell ratio and natalizumab concentration levels in the cerebrospinal fluid of patients with multiple sclerosis. Clin Exp Immunol 180: 383-392, 2015.

13. Dickson DW: Parkinson's disease and parkinsonism: neuropathology. Cold Spring Harb Perspect Med 2: a009258, 2012.

14. Meredith GE and Rademacher DJ: MPTP mouse models of Parkinson's disease: An update. J Parkinsons Dis 1: 19-33, 2011.

15. Luchtman DW, Shao D and Song C: Behavior, neurotransmitters and inflammation in three regimens of the MPTP mouse model of Parkinson's disease. Physiol Behav 98: 130-138, 2009.

16. Deacon RM, Koros E, Bornemann KD and Rawlins JN: Aged $\mathrm{Tg} 2576$ mice are impaired on social memory and open field habituation tests. Behav Brain Res 197: 466-468, 2009. 
17. Chandra G, Rangasamy SB, Roy A, Kordower JH and Pahan K Neutralization of RANTES and eotaxin prevents the loss of dopaminergic neurons in a mouse model of parkinson disease. J Biol Chem 291: 15267-15281, 2016.

18. Krummel MF, Bartumeus F and Gérard A: T cell migration, search strategies and mechanisms. Nat Rev Immunol 16: 193-201, 2016.

19. Walling BL and Kim M: LFA-1 in T cell migration and differentiation. Front Immunol 9: 952, 2018.

20. Miklossy J, Doudet DD, Schwab C, Yu S, McGeer EG and McGeer PL: Role of ICAM-1 in persisting inflammation in Parkinson disease and MPTP monkeys. Exp Neurol 197: 275-283, 2006.

21. Rivera-Nieves J, Gorfu G and Ley K: Leukocyte adhesion molecules in animal models of inflammatory bowel disease. Inflamm Bowel Dis 14: 1715-1735, 2008.
22. Ivanov II, Atarashi K, Manel N, Brodie EL, Shima T, Karaoz U, Wei D, Goldfarb KC, Santee CA, Lynch SV, et al: Induction of intestinal Th17 cells by segmented filamentous bacteria. Cell 139: 485-498, 2009.

23. Ivanov II, Frutos Rde L, Manel N, Yoshinaga K, Rifkin DB, Sartor RB, Finlay BB and Littman DR: Specific microbiota direct the differentiation of IL-17-producing T-helper cells in the mucosa of the small intestine. Cell Host Microbe 4: 337-349, 2008.

This work is licensed under a Creative Commons Attribution-NonCommercial-NoDerivatives 4.0 International (CC BY-NC-ND 4.0) License. 